\section{Mikio OHTSUKA \\ Takako MIURA \\ Toshiyuki YAMAMOTO}

Department of Dermatology,

Fukushima Medical University School

of Medicine, Fukushima, Japan

Reprints: M. Ohtsuka

<motsuka@fmu.ac.jp>

Article accepted on 03/8/2016

\title{
Clinical characteristics, differential diagnosis, and treatment outcome of subcutaneous panniculitis-like T-cell lymphoma: a literature review of published Japanese cases
}

\begin{abstract}
Background: Subcutaneous panniculitis-like T-cell lymphoma (SPTCL) is a rare subtype of non-Hodgkin lymphoma that shows phenotypic features of cytotoxic T cells and preferentially involves subcutaneous tissue. SPTCL is believed to show an indolent clinical course, unless patients develop haemophagocytic syndrome. Various reported therapies include corticosteroids, immunosuppressive drugs, and chemotherapies. The use of chemotherapy as a first-line treatment remains controversial, and treatment approaches for SPTCL have not been established yet. Objectives: To investigate the clinicopathological features, treatment modalities, and outcomes of Japanese patients with SPTCL. Materials \& methods: We performed a literature review of Japanese cases of SPTCL. Results: Twenty-two cases have been reported in the English and Japanese literature. Six cases were excluded due to a lack of sufficient clinical and immunohistological data, and treatment modalities and outcome were available in 16 cases. Clinical characteristics of the Japanese cases were generally similar to those of Western countries. Approximately half of the Japanese patients were treated initially with corticosteroids. Among them, more than half subsequently received chemotherapies due to a lack of response or recurrence of the disease. Overall, chemotherapy was used for approximately $80 \%$ of reported cases as a primary or secondary therapy. Conclusion: Our findings suggest that corticosteroids may be beneficial for some SPTCL patients, but most patients required chemotherapy during the course of their disease. Further investigations are needed to both establish appropriate treatment strategies for SPTCL and clarify predictive factors in order to identify patients who may benefit from corticosteroid therapy as a primary treatment.
\end{abstract}

Key words: chemotherapy, corticosteroid, immunosuppressive drug, Japanese case, subcutaneous panniculitis-like T-cell lymphoma
$\mathrm{T}$ here are various subtypes of cutaneous lymphomas, each with different clinical manifestations, histological features, and prognosis. Mycosis fungoides is the most common and well characterised subtype, accounting for more than $50 \%$ of all cutaneous lymphomas $[1,2]$. Subcutaneous panniculitis-like T-cell lymphoma (SPTCL) is a rare subtype of cutaneous lymphoma that preferentially involves subcutaneous tissue. Since its first description by Gonzales et al. in 1991, as a peculiar lymphoma mimicking panniculitis [3], many cases have been reported under the diagnosis of subcutaneous lymphoma, which showed various phenotypes of neoplastic lymphoid cells, such as CD4+CD8-, CD4-CD8+, or CD4CD8-CD56+, with diverse clinical courses ranging from indolent to highly aggressive. In the WHO-EORTC classification of cutaneous lymphomas proposed in 2005 [1], SPTCL was defined as CD8+ cytotoxic T-cell lymphoma expressing alpha/beta T-cell receptors (TCRs) that are confined to subcutaneous fat. According to this definition, SPTCL generally show indolent clinical behaviour, in contrast to other lymphomas involving subcutaneous tissue, such as gamma/delta T-cell lymphoma or NK cell lymphoma.

In European countries, SPTCL was estimated to account for $1 \%$ of cutaneous lymphoma cases [1], and the clinicopathological features, treatment modalities, and clinical outcomes of European cases were reported by Willemze et al. in 2008 [4]. In Japan, the result of the nationwide study on cutaneous lymphomas from 2007 to 2011 revealed the prevalence of SPTCL to be $2.3 \%$ of cutaneous lymphoma cases [2]. However, the clinicopathological features of Japanese patients with SPTCL have not yet been well characterised. Therefore, we performed a literature review in order to investigate the clinicopathological features, 
therapeutic modalities, and treatment outcomes of Japanese SPTCL cases, and to propose appropriate strategies for management of SPTCL.

\section{Methods}

We searched for articles using PubMed and the Japanese medical literature database provided by the Japan Medical Abstract Society. We used the term "subcutaneous panniculitis-like T-cell lymphoma" and its directly translated Japanese form for searching on PubMed and the Japanese medical literature database, respectively. We retrieved all articles of Japanese cases, excluding those described in meeting abstracts.

\section{Results}

\section{Clinical and histological features}

We identified 22 Japanese cases of SPTCL reported in the literature. Among them, we excluded six cases due to lack of sufficient clinical and immunohistological data to unequivocally diagnose the cases as SPCTL. Finally, we retrieved 16 Japanese cases of SPTCL [5-19], and the clinical and immunophenotypic features are summarised in (tables 1-3). Eleven of these cases were reported in English language journals and the remaining five cases were obtained from Japanese journals. The cases consisted of five males and 11 females, with ages ranging from one to 73 years old (average: 32.1 years old). All patients had subcutaneous nodules, which were most frequent on the lower legs (figure 1A). B symptoms, such as fever, night sweats, and weight loss were observed in 13 of 16 cases. Autoimmune disorders were present in two cases, including Sjögren's syndrome and type 1 diabetes mellitus. Laboratory abnormalities, including leukocytopenia and liver dysfunction, were common, occurring in approximately 50\% of cases. The existence of haemophagocytic syndrome (HPS) was mentioned in 11 cases. Among them, HPS was observed in five cases. Histologically, all 16 cases showed mild to moderate lobular infiltration of lymphoid cells in subcutaneous fat tissue with only scant lymphoid infiltrates in the dermis (figures $1 B, C$ ). All cases tested were positive for CD3 and CD8, and negative for CD4 and CD56. Cytotoxic molecules, such as T-cell intracellular antigen (TIA)-1 and granzyme B, were positive in all cases tested. In addition, TCR beta chain expression was detected in all eight cases examined by immunohistochemistry (figures $1 D-F$ ), and clonal rearrangement of TCR genes was confirmed in 10 out of 11 cases tested, either by Southern blot analysis or polymerase chain reaction. Epstein-Barr (EB) virus-encoded small nuclear RNA (EBER) was detected in one of 12 cases tested by in situ hybridisation.

\section{Treatment modalities and clinical outcome}

The treatment modalities and outcomes are summarised in figure 2 and tables 1 , 4. One patient showed spontaneous remission (Case 16). Seven patients were treated with corticosteroids or immunosuppressive drugs as a

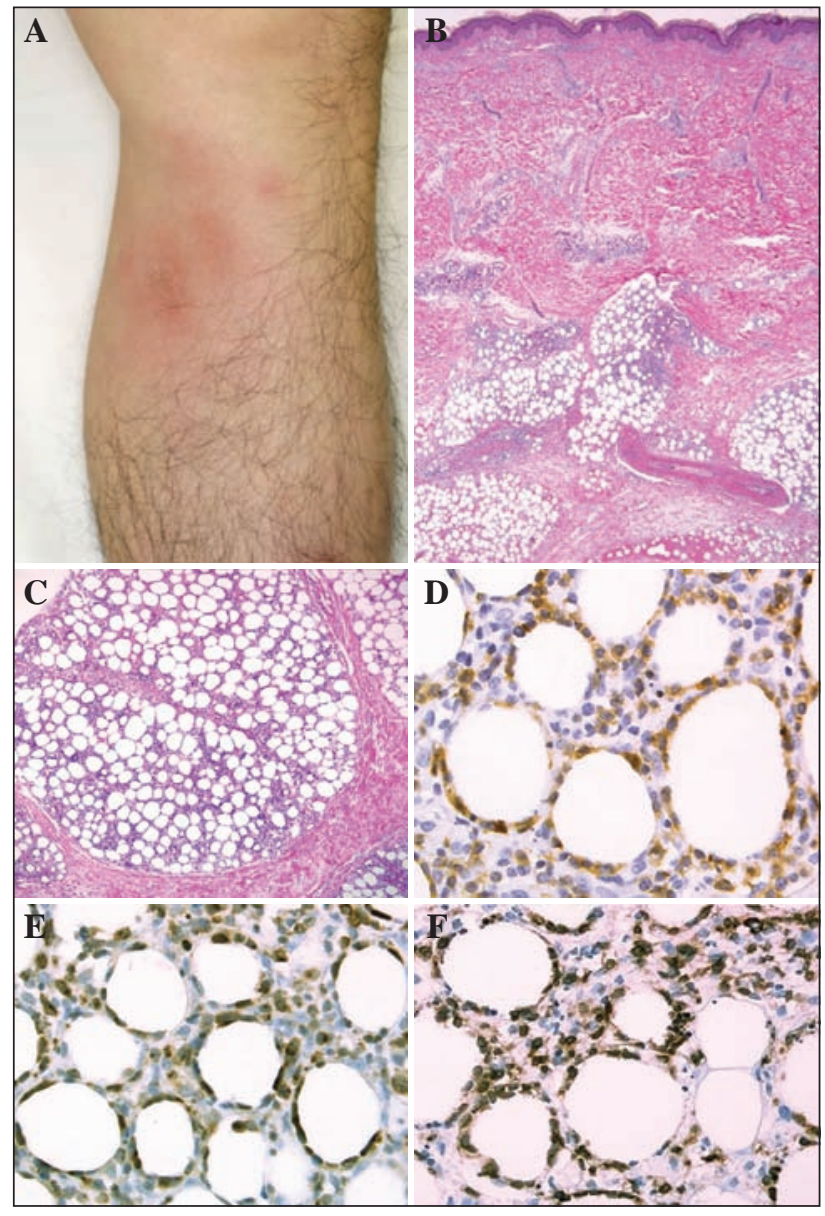

Figure 1. Representative case of subcutaneous panniculitislike T-cell lymphoma. A) Erythematous subcutaneous nodules are seen at the popliteal region. B) Lymphoid cell infiltration is almost confined to subcutaneous tissue (haematoxylin-eosin stain; original magnification: $\times 40$ ). C) Lobular infiltration of lymphoid cells that surround individual adipocytes is seen (haematoxylin-eosin stain; original magnification: $\times 100$ ). DF) Immunostaining for CD8 (D), granzyme B (E), and beta-F1 (F). The infiltrating lymphoid cells are $\mathrm{CD} 8$, granzyme $\mathrm{B}$, and beta-F1 positive (original magnification: $\times 200$ ).

first-line treatment (non-chemotherapy group). Of these seven patients, three achieved complete remission (CR) without the use of chemotherapy, and four patients, including two complicated by HPS, were treated with chemotherapy consisting of cyclophosphamide, doxorubicin, vincristine, and prednisone (CHOP) or CHOP-like regimens as a second-line treatment for the unresponsive or recurrent lesions. Among the latter four patients, two were further treated with high-dose chemotherapy supported by haematopoietic stem cell transplantation. All patients in the non-chemotherapy group were in remission at the time their cases were reported in the literature. Eight patients were initially treated with chemotherapy (chemotherapy group), which consisted of a CHOP or CHOP-like regimen for seven patients, and hyper-CAVD (cyclophosphamide, vincristine, doxorubicin, dexamethasone, methotrexate, and cytarabine) for one patient. Three of these patients achieved $\mathrm{CR}$, one patient died due to progression of the disease, and one patient died of an unrelated cause during 


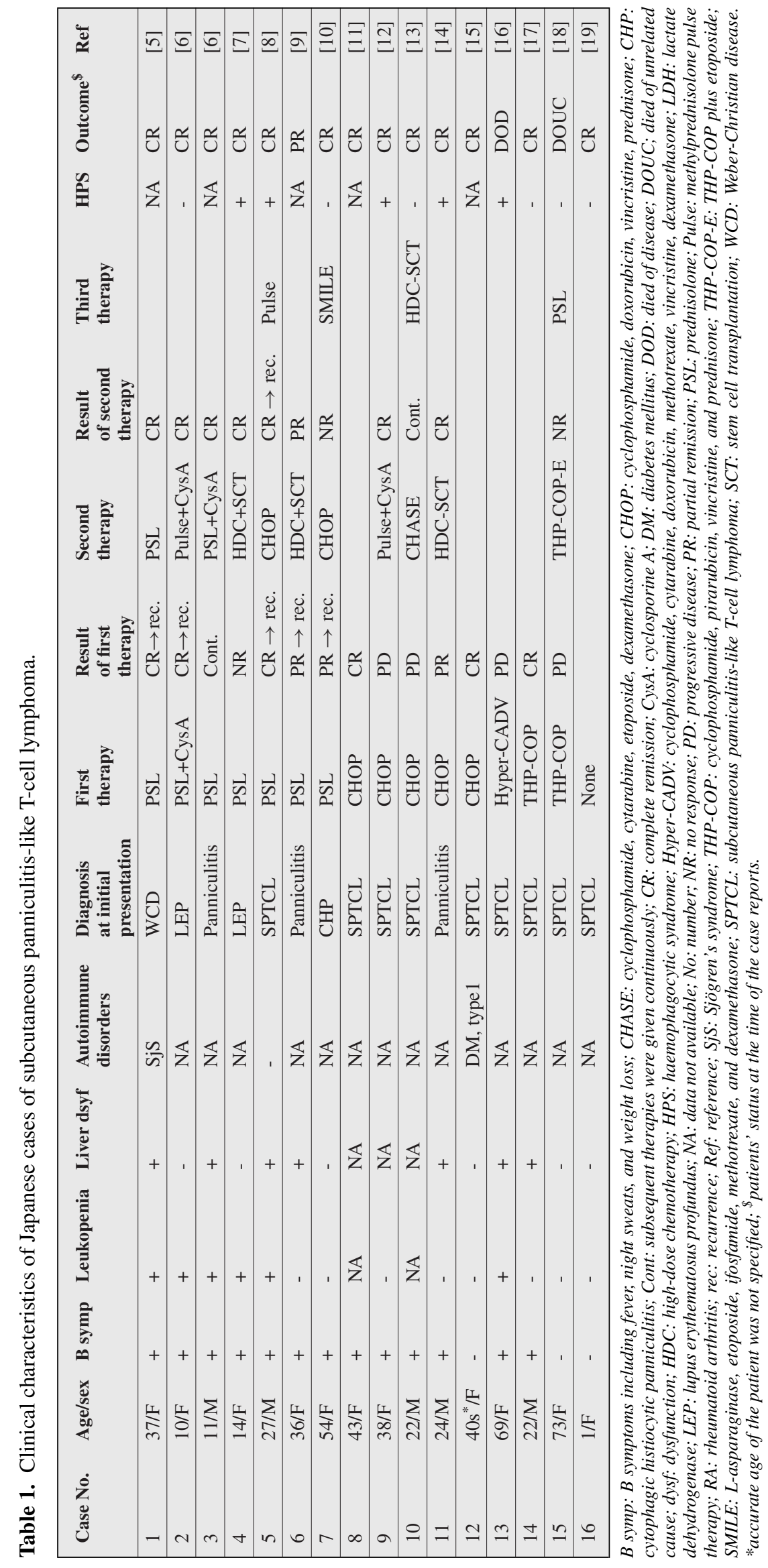


Table 2. Immunohistochemical features of Japanese cases of subcutaneous panniculitis-like T-cell lymphoma.

\begin{tabular}{|c|c|c|c|c|c|c|c|c|c|}
\hline Case No. & CD3 & CD4 & CD8 & CD56 & TIA-1 & GranB & $\beta$ F1 & EBER & Clonality \\
\hline 1 & + & NA & + & - & + & + & + & - & + \\
\hline 2 & + & - & + & - & + & + & NA & NA & + \\
\hline 3 & + & NA & + & NA & + & NA & + & NA & + \\
\hline 4 & + & - & + & - & + & + & + & - & NA \\
\hline 5 & + & - & + & - & + & + & + & - & + \\
\hline 6 & + & - & + & - & + & + & NA & - & NA \\
\hline 7 & + & - & + & - & NA & + & NA & - & + \\
\hline 8 & NA & - & + & - & NA & NA & + & NA & NA \\
\hline 9 & + & - & + & - & NA & + & + & - & NA \\
\hline 10 & + & - & + & - & + & + & NA & - & + \\
\hline 11 & + & - & + & - & + & + & NA & - & + \\
\hline 12 & + & - & + & - & NA & + & + & NA & NA \\
\hline 13 & + & - & + & - & NA & + & NA & + & + \\
\hline 14 & + & - & + & - & + & + & NA & - & + \\
\hline 15 & + & - & + & - & + & NA & NA & - & + \\
\hline 16 & + & - & + & - & + & + & + & - & oligo \\
\hline Total & $15 / 15$ & $0 / 14$ & $16 / 16$ & $15 / 15$ & $11 / 11$ & $13 / 13$ & $8 / 8$ & $1 / 12$ & $10 / 11$ \\
\hline
\end{tabular}

EBER: Epstein-Barr virus-encoded small nuclear RNA; GranB: granzyme B; NA: data not available; oligo: oligoclonal; TIA-1: T-cell intracellular antigen-1.

second-line chemotherapy. Among the remaining three patients, two received high-dose chemotherapy with stem cell transplantation, and one was treated with a corticosteroid in combination with cyclosporine A. These three patients resulted in remission. In the chemotherapy group, six achieved remission, one died of lymphoma progression, and one died of an unrelated cause at the time of the last follow-up visit. Of the two fatal cases, one had associated HPS.

Regarding patients with associated HPS, all five patients in our study received chemotherapy as a first-line or a second-line treatment. Of the two patients belonging to the non-chemotherapy group, one had HPS at the time of initial corticosteroid therapy and failed to respond to prednisolone. The patient subsequently received high-dose chemotherapy, which induced remission of his disease (Case 4). The other patient developed HPS after the initial prednisolone and subsequent CHOP therapy, and HPS was successfully treated with methylprednisolone pulse therapy (Case 5). Of the three patients belonging to the chemotherapy group, one patient developed HPS after failure of the initial CHOP therapy, and was then treated with methylprednisolone pulse therapy in combination with cyclosporine A (Case 9). One patient with HPS at the time of initial diagnosis failed to respond to CHOP and received high-dose chemotherapy supported by stem cell transplantation (Case 11). These

Table 3. Clinical features of patients with SPTCL.

\begin{tabular}{|c|c|c|c|c|}
\hline & Japanese patients* & European patients $\$$ & Chinese patients ${ }^{\&}$ & Korean patients ${ }^{\&}$ \\
\hline Number of patients & 16 & 63 & 22 & 14 \\
\hline Mean age (range) & 32.1 years $^{\dagger}(1-73)$ & 36 years $(7-79)$ & 32 years $(9-72)$ & 35 years $(7-73)$ \\
\hline Male:female ratio & $5: 11$ & $21: 42$ & $8: 14$ & $10: 4$ \\
\hline B symptoms & $13 / 16(81 \%)$ & $37 / 63(59 \%)$ & $8 / 22(36 \%)$ & $9 / 13(69 \%)$ \\
\hline Autoimmune disease & $2 / 16^{\S}(13 \%)$ & $12 / 63(19 \%)$ & NA & NA \\
\hline Leukopenia & $6 / 14(43 \%)$ & & NA & $7 / 14(50 \%)$ \\
\hline Liver dysfunction & $7 / 13(54 \%)$ & $29 / 63(46 \%)^{\#}$ & NA & NA \\
\hline Increased LDH & $12 / 16(75 \%)$ & & NA & $9 / 13(69 \%)$ \\
\hline HPS & $5 / 11(45 \%)$ & $11 / 63(17 \%)$ & NA & $2 / 14(14 \%)$ \\
\hline Clonality & 10/11 (91\%) & $37 / 45(82 \%)$ & $15 / 15(100 \%)$ & $4 / 8(50 \%)$ \\
\hline EBER & $1 / 12(8 \%)$ & $0 / 38(0 \%)$ & $1 / 22(5 \%)$ & $0 / 8(0 \%)$ \\
\hline Death & $2 / 16(13 \%)$ & $9 / 63(14 \%)$ & $5 / 22(22 \%)$ & $2 / 14(14 \%)$ \\
\hline
\end{tabular}

B symptoms: fever, night sweats, and weight loss; NA: data not available; EBER: Epstein-Barr virus-encoded small nuclear RNA; HPS: haemophagocytic syndrome. ${ }^{\dagger}$ excluding one patient whose accurate age was not specified (Case 12 in table 1); ${ }^{*}$ data from reference [4]; ${ }^{\$}$ data from reference [22]; \& data

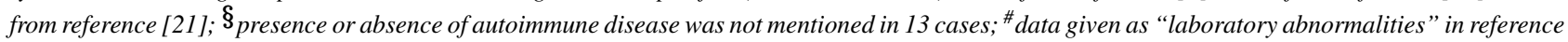
[4]. 


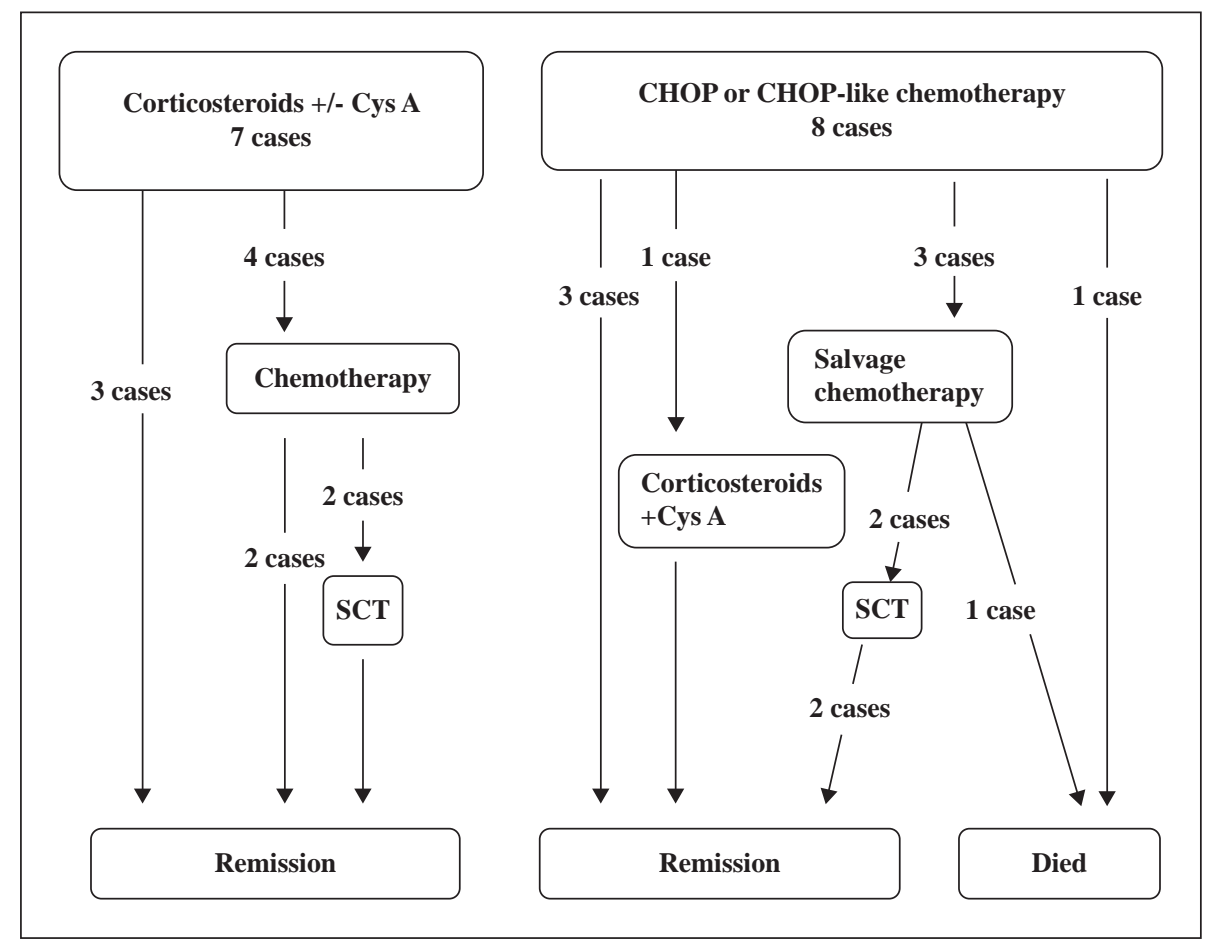

Figure 2. Clinical course of Japanese patients with subcutaneous-panniculitis-like T-cell lymphoma. Seven patients were treated with corticosteroids or immunosuppressive drugs as a first-line treatment. All of these patients were in remission at the time their cases were reported in the literature. Eight patients were initially treated with chemotherapy. Among them, six patients were in remission, one patient died due to the progression of lymphoma, and one patient died of an unrelated cause. $\mathrm{CHOP}$ : cyclophosphamide, doxorubicin, vincristine, and prednisone; CysA: cyclosporine A; SCT: stem cell transplantation.

two patients were in remission at the time the case reports were published. Another patient died of rapidly progressive disease despite chemotherapy, and HPS was revealed by an autopsy (Case 13).

\section{Clinical characteristics of patients treated with corticosteroids and chemotherapy}

We next compared the clinical characteristics of the non-chemotherapy group with those of the chemotherapy group (table 5). In both groups, the male to female ratio and the incidence of HPS were almost identical, and clonal rearrangement of TCR genes was detected in all cases. Compared with the chemotherapy group, patients in the non-chemotherapy group were younger and had more frequent B symptoms and leukocytopenia, but liver dysfunction and increased lactate dehydrogenase levels were more common in the chemotherapy group. Autoimmune disease was observed in one case in both groups. In the chemotherapy group, most cases were diagnosed with SPTCL at initial presentation. In contrast, only one patient was initially diagnosed with SPTCL in the nonchemotherapy group, and the other patients were tentatively diagnosed with unspecified panniculitis and lupus profundus in two cases, respectively, and cytophagic histiocytic panniculitis and Weber-Christian disease in one case. Two fatal cases, including one EBER-positive case and one case associated with HPS, were included in the chemotherapy group.

\section{Discussion}

SPTCL is a rare form of cutaneous T-cell lymphoma that preferentially involves subcutaneous tissue. The diagnostic criteria and clinical features of SPTCL described in the WHO classification were established based on patient data collected mainly from European countries [4, 20]. Because the frequencies of lymphoma subtypes are known to vary among racial groups, it is important to investigate the clinical characteristics and treatment outcome of Japanese SPTCL cases.

Our findings demonstrated that the clinical characteristics of Japanese cases were almost identical to European cases, such as younger age at onset (relative to other cutaneous T-cell lymphomas), twofold predominance of females over males, high frequency of laboratory abnormalities, and low mortality rate of less than $15 \%$ [4]. In addition, the initial therapeutic modalities were almost identical in both the Japanese and European cases. B symptoms were more common in Japanese cases, whereas autoimmune disorders were more frequent in European cases. The clinical characteristics of the Japanese cases were also similar to those from Chinese and Korean cases, except for a male predominance in Korean cases [21], as well as a low incidence of B symptoms and high mortality rate in Chinese cases [22] (table 3). These differences in clinical characteristics among different racial groups may be due to small sample size of the Asian cases. It is notable that EBER was detected in one Japanese patient and one Chinese patient 
Table 4. Treatment modalities and outcomes of patients with SPTCL.

\begin{tabular}{|c|c|c|}
\hline & $\begin{array}{l}\text { Japanese } \\
\text { patients }\end{array}$ & $\begin{array}{l}\text { European } \\
\text { patients* }\end{array}$ \\
\hline Number of patients & 16 & 63 \\
\hline \multicolumn{3}{|l|}{ Initial treatment } \\
\hline $\begin{array}{l}\text { Corticosteroids/ } \\
\text { immunosuppressive drugs }\end{array}$ & $7(44 \%)$ & $24(38 \%)$ \\
\hline $\begin{array}{l}\text { CHOP or CHOP-based } \\
\text { chemotherapy }\end{array}$ & $8(50 \%)$ & $31(49 \%)$ \\
\hline Radiation & $8(50 \%)$ & $3(5 \%)$ \\
\hline Surgery & $0(0 \%)$ & $2(3 \%)$ \\
\hline No treatment & $1(6 \%)$ & $2(3 \%)$ \\
\hline \multicolumn{3}{|l|}{$\begin{array}{l}\text { Complete remission after } \\
\text { initial treatment }\end{array}$} \\
\hline $\begin{array}{l}\text { Corticosteroids/ } \\
\text { immunosuppressive drugs }\end{array}$ & $3 / 7(43 \%)$ & $16 / 24(67 \%)$ \\
\hline $\begin{array}{l}\text { CHOP or CHOP-based } \\
\text { chemotherapy }\end{array}$ & $3 / 8(38 \%)$ & $19 / 31(62 \%)$ \\
\hline $\begin{array}{l}\text { Number of patients treated } \\
\text { with chemotherapy }\end{array}$ & $12 / 16(75 \%)$ & $39 / 62(63 \%)$ \\
\hline \multicolumn{3}{|l|}{ Status at the time of the report } \\
\hline Alive without disease & $13(82 \%)$ & $39(62 \%)$ \\
\hline Alive with disease & $1(6 \%)$ & $15(24 \%)$ \\
\hline Death & $2^{\#}(12 \%)$ & $9(14 \%)$ \\
\hline
\end{tabular}

CHOP: cyclophosphamide, doxorubicin, vincristine, prednisone. $\dagger$ including both first-line and second-line therapies; \#including one patient who died of an unrelated cause; *data from reference [4].

by in situ hybridisation. The Japanese patient died due to disease progression and the Chinese patient was in good health at the time of the last follow-up visit. SPTCL is generally believed to be negative for latent EB-virus infection $[1,4,20]$. However, EB virus-associated lymphoid malignancies, such as extranodal NK/T-cell lymphoma and Burkitt lymphoma [1,20], are well known to be prevalent in Asian countries such as Japan and China, which is most likely due to genetic susceptibility. Therefore, EBER positivity alone cannot exclude the possibility of SPTCL in regions where EB virus-associated lymphoid malignancies are prevalent, although thorough investigations including immunohistochemical analysis, gene rearrangement assays, and systemic work-up are mandatory before establishing the diagnosis of SPTCL in EBER-positive cases.

The most important and controversial issue in the treatment of SPTCL is whether SPTCL should be treated with chemotherapy or whether it can be managed with corticosteroids or immunosuppressive drugs. Among the various treatment guidelines for cutaneous lymphomas, including those of the British Association of Dermatology [23], the European Organisation for Research and Treatment of Cancer consensus recommendation [24], the National Comprehensive Cancer Network clinical practice guidelines [25], and the European Society for Medical Oncology (ESMO) guidelines [26], only the latter guidelines provide a treatment recommendation for SPTCL. In the ESMO guidelines, corticosteroids or immunosuppressive drugs are recommended as a first-line treatment for patients without HPS, and chemotherapy is indicated as an optional therapy in cases refractory to immunosuppressive therapy or in cases with HPS [26]. The Japanese guidelines for
Table 5. Clinical characteristics of patients who received corticosteroids and chemotherapy treatment.

\begin{tabular}{|lll|}
\hline & $\begin{array}{l}\text { Corticosteroids/ } \\
\text { immunosuppressive } \\
\text { drugs }\end{array}$ & Chemotherapy \\
\hline Number of patients & 7 & 8 \\
\hline Mean age (range) & 27 years (10-54) & 41.6 years $^{\dagger}(22-73)$ \\
\hline Male:female ratio & $2: 5$ & $3: 5$ \\
\hline B symptoms & $7 / 7(100 \%)$ & $6 / 8(75 \%)$ \\
\hline Leukocytopenia & $5 / 7(71 \%)$ & $1 / 6(17 \%)$ \\
\hline Liver dysfunction & $4 / 7(57 \%)$ & $3 / 5(60 \%)$ \\
\hline Increased LDH & $5 / 7(71 \%)$ & $7 / 8(88 \%)$ \\
\hline Clonality & $5 / 5(100 \%)$ & $5 / 5(100 \%)$ \\
\hline HPS & $2 / 4(50 \%)$ & $3 / 6(50 \%)$ \\
\hline Autoimmune disease & $1 / 7(14 \%)$ & $1 / 8(13 \%)$ \\
\hline Initial diagnosis & & \\
\hline SPTCL & $1 / 7(14 \%)$ & $7 / 8(88 \%)$ \\
Panniculitis & $2 / 7(29 \%)$ & $1 / 8(13 \%)$ \\
Lupus profundus & $2 / 7(29 \%)$ & $0 / 10(0 \%)$ \\
Other & $2 / 7^{\#}(29 \%)$ & $0 / 10(0 \%)$ \\
\hline Death & $0 / 7(0 \%)$ & $2 / 8(25 \%)$ \\
\hline
\end{tabular}

B symptoms: fever, night sweats, and weight loss; HPS: haemophagocytic syndrome; LDH: lactate dehydrogenase; SPTCL: subcutaneous panniculitis-like T-cell lymphoma; TCR: T-cell receptor. ${ }^{\dagger}$ excluding one patient whose accurate age was not specified (Case 12 in table 1); ${ }^{\text {\# }}$ one case of cytophagic histiocytic panniculitis and one case of Weber-Christian disease.

cutaneous lymphoma recommend similar treatment approaches [27]. The ESMO recommendation regarding treatment options for SPTCL might have been made mainly based on the report of Willemze et al. in which the clinicopathological features and treatment outcomes of 63 European cases of SPTCL were investigated [4]. In their report, 55 patients received systemic therapy, which consisted of chemotherapy for 31 patients (chemotherapy group) and corticosteroids or immunosuppressive drugs for 24 patients (non-chemotherapy group). Although the response rate of the initial therapies was higher in the non-chemotherapy group (16 CR and five partial response [PR]; response rate: $88 \%$ ) than in the chemotherapy group (19 CR and three PR; response rate: $71 \%$ ) (table 4), the remission rate at the time of the last follow-up visit was similar between the two groups (non-chemotherapy group: 58\%; chemotherapy group: $61 \%$ ). These findings suggested that patients with SPTCL may benefit equally from chemotherapy and corticosteroids/immunosuppressive drugs. Therefore, the authors concluded that their findings do not support the routine use of multi-agent chemotherapy as a first-line treatment for patients with SPTCL. However, the relapse of complete responders was more frequent in the non-chemotherapy group $(9 / 19 ; 56 \%)$ than in the chemotherapy group $(2 / 19$; $10 \%$ ), and eight of 24 patients in the non-chemotherapy group received chemotherapy for unresponsive or recurrent lesions, suggesting that corticosteroids or immunosuppressive drugs may not have sufficient effect on some patients with SPTCL.

In contrast to European cases, most of the reported Chinese and Korean cases were treated with chemotherapy $[21,22]$. Fourteen of 18 Chinese patients (78\%) received 
chemotherapy, and nine patients responded (six CR and three PR; response rate: 64\%). The other four patients were treated with Chinese herbal medicine or surgical excision of the solitary lesion, leading to a durable response for all of these patients. Ten of 14 Korean patients (71\%) were treated with chemotherapy, three received immunosuppressive therapy, and one received only supportive care for the patient's symptoms. Of the ten patients receiving chemotherapy, five achieved remission (four CR and one PR; response rate: 50\%). Of the three patients treated with immunosuppressive therapy, only one patient responded. Accordingly, chemotherapy was beneficial in approximately two thirds of the Chinese and Korean patients, whereas therapeutic benefit of corticosteroids or immunosuppressive drugs was uncertain because the number of patients treated with corticosteroids was very small.

In Japan, the number of patients initially treated with corticosteroids or immunosuppressive drugs and those treated with chemotherapy was almost the same, and the response rate of the initial therapy did not show remarkable difference between the two treatment groups (table 4). Most patients who had been initially diagnosed with SPTCL received chemotherapy as a first-line treatment, whereas the majority of patients who were initially diagnosed with inflammatory panniculitis and then later diagnosed with SPTCL were treated with corticosteroids (table 5). This suggests that the initial treatment modalities have been determined depending on the initial diagnosis in most Japanese cases. Since SPTCL often simulates various inflammatory diseases, such as erythema nodosum, lupus profundus, and other miscellaneous disorders manifesting with panniculitis, a clear distinction between SPTCL and these panniculitic disorders is often difficult at the initial presentation [2830]. Thus, we consider that corticosteroid treatment may be justified for patients with lesions suggestive, but not diagnostic, of SPTCL, especially if they have an accompanying constitutional symptom. However, four of seven Japanese cases in the non-chemotherapy group needed chemotherapy for unresponsive or recurrent lesions, indicating that the therapeutic effect of corticosteroid treatment for SPTCL may be limited to a certain subset of patients, and we cannot predict exactly which patients could benefit from corticosteroid therapy. Previous reports have shown that re-administration of corticosteroids or immunosuppressive drugs can induce remission of recurrent lesions that developed after withdrawal of initial corticosteroid therapy $[4,5,31]$. Therefore, re-administration of these drugs may be a therapeutic option, instead of chemotherapy, at least until disease progression for relapsed patients, especially if they do not suffer from HPS. However, duration of remission was reported to be short, and disease progression typically occurred once steroid tapering was initiated [32]. Thus, we believe that chemotherapy should be considered immediately when relapsed patients do not respond to re-administration of corticosteroids or immunosuppressive drugs.

Another important issue is how SPTCL patients complicated by HPS should be treated. Five patients included in our study developed HPS at various stages during the course of the disease and received various therapies; two patients who failed to respond to conventional-dose chemotherapy received high-dose chemotherapy and resulted in remission (Cases 4 and 11), two patients were successfully treated with methylprednisolone pulse therapy either alone or in combination with cyclosporine A (Cases 5 and 9), and one patient who received conventional-dose chemotherapy died of disease progression (Case 13). In our cases associated with HPS, none responded to conventional-dose chemotherapy. These results are in accordance with the previous view that $\mathrm{CHOP}$ or CHOP-like therapy is not very successful for SPTCL associated with HPS [4], and methylprednisolone pulse therapy, either alone or in combination with immunosuppressive drugs, can be effective for patients associated with HPS [33-35]. In addition, highdose chemotherapy, followed by stem cell transplantation, has been suggested to be an important option for patients associated with HPS [4, 36, 37].

At present, it still remains unclear whether chemotherapy is actually more beneficial than corticosteroid treatment for SPTCL, however, based on the result of our review of Japanese SPTCL cases, we believe that chemotherapy may be preferred for patients who have been diagnosed unequivocally with SPTCL. In such cases, corticosteroid therapy may be acceptable as an alternative treatment option, especially in cases without HPS, however, careful follow-up is indispensable and chemotherapy should be considered when a sufficient effect cannot be achieved by corticosteroids or immunosuppressive drugs. For patients associated with HPS, methylprednisolone pulse therapy or high-dose chemotherapy, followed by stem cell transplantation, may possibly be preferred over conventional-dose chemotherapy.

A limitation of this article is that the patients' data were collected from published reports, making it difficult to disclose the long-term outcome of each patient. Further investigations are needed in order to establish appropriate treatment strategies for SPTCL, and to clarify the predictive factors which could be used to identify patients who may benefit from corticosteroid therapy as a first-line treatment.

Disclosure. Financial support: none. Conflict of interest: none.

\section{References}

1. Willemze R, Jaffe ES, Burg G, et al. WHO-EORTC classification for cutaneous lymphomas. Blood 2005; 105: 3768-85.

2. Hamada T, Iwatsuki K. Cutaneous lymphoma in Japan: a nationwide study of 1733 patients. J Dermatol 2014;41:3-10.

3. Gonzalez CL, Medeiros L, Braziel RM, Jaffe ES. T-cell lymphoma involving subcutaneous tissue. A clinicopathologic entity commonly associated with hemophagocytic syndrome. Am J Surg Pathol 1991; 15: 17-27.

4. Willemze $R$, Jansen $P M$, Cerroni $L$, et al. EORTC Cutaneous Lymphoma Group. Subcutaneous panniculitis-like T-cell lymphoma: definition, classification, and prognostic factors: an EORTC Cutaneous Lymphoma Group Study of 83 cases. Blood 2008; 111 : 838-45.

5. Yokota K, Akiyama Y, Adachi D, et al. Subcutaneous panniculitislike T-cell lymphoma accompanied by Sjögren's syndrome. Scand J Rheumatol 2009; 38: 494-5.

6. Nagai K, Nakano N, Iwai $T$, et al. Pediatric subcutaneous panniculitis-like T-cell lymphoma with favorable result by immunosuppressive therapy: a report of two cases. Pediatr Hematol Oncol 2014;31:528-33. 
7. Sakurai E, Satoh T, Akiko YA, et al. Subcutaneous panniculitislike T-cell lymphoma (SPTCL) with hemophagocytosis (HPS): successful treatment using high-dose chemotherapy (BFM-NHL \& ALL-90) and autologous peripheral blood stem cell transplantation. J Clin Exp Hematop 2013; 53: 135-40.

8. Miura $T$, Kawakami $Y$, Sato $M$, Ohtsuka $M$, Yamamoto $T$. Hemophagocytic syndrome occurred in a patient with subcutaneous panniculitis-like T-cell lymphoma without overt skin lesion: successful treatment with steroid pulse therapy. J Dermatol 201 1;38: $1113-5$

9. Sugita K, Kobayashi M, Hino R, Ohta T, Ohshima K, Tokura Y. A Japanese case of typical Epstein-Barr virus non-associated subcutaneous panniculitis-like T-cell lymphoma. J Dermatol 2004; 31: 253-4.

10. Muramatsu S, Nishida E, Nishio E, Morita A. A case of subcutaneous panniculitis-like T-cell lymphoma. Practical Dermatol 2014; 36: e63-6.

11. Mitsuhashi K, Momose $M$, Masuda A, Tsunemi $Y$, Motoji T. Positron emission tomography revealed diffuse involvement of the lower legs and occult extracutaneous lesions in subcutaneous panniculitis-like T-cell lymphoma. Clin Nucl Med 2013; 38: 209-11.

12. Mizutani $S$, Kuroda J, Shimura $Y$, et al. Cyclosporine $A$ for chemotherapy-resistant subcutaneous panniculitis-like T cell lymphoma with hemophagocytic syndrome. Acta Haematol 2011 ; 126: 8-12.

13. Nakahashi $H$, Tsukamoto $N$, Yamane $A$, et al. Autologous peripheral blood stem cell transplantation to treat $\mathrm{CHOP}$-refractory aggressive subcutaneous panniculitis-like $\mathrm{T}$ cell lymphoma. Acta Haematol 2009; 121:239-42.

14. Mukai HY, Okoshi Y, Shimizu S, et al. Successful treatment of a patient with subcutaneous panniculitis-like T-cell lymphoma with high-dose chemotherapy and total body irradiation. Eur J Haematol 2003; 70: 413-6

15. Gondo C, Osada M, Yamamoto $H$, et al. A typical case of subcutaneous panniculitis-like T-cell lymphoma. Shindan Byori 2011;28: 149-51.

16. Ohnishi A, Yoshida Y, Yamamoto O, Hayashi K. Aggressive subcutaneous panniculitis-like T-cell lymphoma. Nishinihon J Dermatol 2009; $71: 275-7$.

17. Ishii $Y$, Nishida T, Amano M, Setoyama M, Yamashita K, Kitamura T. A case of subcutaneous panniculitis-like T-cell lymphoma (SPTCL) with multiple subcutaneous nodules on the trunk and lower limbs. Skin Cancer 2008; 23: 130-3

18. Osawa R, Yokota K, Sawamura D, et al. A case of subcutaneous T-cell lymphoma. Jap J Clin Dermatol 2005; 59: $1127-9$.

19. Kawachi $Y$, Furuta J, Fujisawa $Y$, Nakamura $Y$, Ishii $Y$, Otsuka F. Indolent subcutaneous panniculitis-like T cell lymphoma in a 1-year-old child. Pediatr Dermatol 2012; 29: 374-7.

20. Swerdlow SH, Campo E, Harris NS, et al. WHO Classification of Tumours of Haematopoietic and Lymphoid Tissues, $4^{\text {th }}$ Ed.. Lyon: IARC Press, 2008, 294-295.

21. Lee DW, Yang JH, Lee $S M$, et al. Subcutaneous panniculitis-like Tcell lymphoma: a clinical and pathologic study of 14 Korean patients. Ann Dermatol 2011 ; 23: 329-37.

22. Kong YY, Dai B, Kong JC, et al. Subcutaneous panniculitis-like Tcell lymphoma: a clinicopathologic, immunophenotypic, and molecular study of 22 Asian cases according to WHO-EORTC classification. Am J Surg Pathol 2008; 32: 1495-502.
23. Whittaker SJ, Marsden JR, Spittle $M$, Russell Jones R. Joint British Association of Dermatologists and U.K Cutaneous Lymphoma Group guidelines for the management of primary cutaneous T-cell lymphomas. Br J Dermatol 2003; 149: 1095-107.

24. Trautinger $F$, Knobler $R$, Willemze $R$, et al. EORTC consensus recommendations for the treatment of mycosis fungoides/Sézary syndrome. Eur J Cancer 2006; 42: 1014-30

25. Zelenetz AD, Abramson JS, Advani RH, et al. NCCN Clinical Practice Guidelines in Oncology: non-Hodgkin's lymphomas. J Natl Compr Canc Netw 2010; 8: 288-334.

26. Willemze R, Hodak E, Zinzani PL, Specht L, Ladetto L. M ESMO Guidelines Working Group Primary cutaneous lymphomas: ESMO Clinical Practice Guidelines for diagnosis, treatment and follow-up. Ann Oncol 2013; 24: 49-54

27. Sugaya $M$, Hamada $T$, Kawai $K$, et al. Guidelines for the management of cutaneous lymphomas (2011): a consensus statement by the Japanese Skin Cancer Society - Lymphoma Stukdy Group. J Dermatol 2013; 40: 2-14

28. Arps DP, Patel RM. Lupus profundus (panniculitis): a potential mimic of subcutaneous panniculitis-like T-cell lymphoma. Arch Pathol Lab Med 2013; 137: 1211-5.

29. Pincus LB, LeBoit $P E, M c C a l m o n t ~ T H$, et al. Subcutaneous panniculitis-like T-cell lymphoma with overlapping clinicopathologic features of lupus erythematosus: coexistence of 2 entities? Am J Dermatopathol 2009; 31: 520-6.

30. Bosisio F, Boi S, Caputo V, et al. Lobular panniculitic infiltrates with overlapping histopathologic features of lupus panniculitis (lupus profundus) and subcutaneous T-cell lymphoma: a conceptual and practical dilemma. Am J Surg Pathol 2015; 39: 206-1 1 .

31. Go SI, Lee WS, Kang MH, Kim IS, Kim DC, Lee JH, Cyclosporine $\mathrm{JH}$. A treatment for relapsed subcutaneous panniculitis-like T-cell lymphoma: a case with long-term follow-up. Korean J Hematol 2012; 47: 146-9.

32. Go RS, Wester SM. Immunophenotypic and molecular features, clinical outcomes, treatments, and prognostic factors associated with subcutaneous panniculitis-like T-cell lymphoma: a systematic analysis of 156 patients reported in the literature. Cancer 2004; 101: 1404-13.

33. Tsukamoto $Y$, Katsunobu $Y$, Omura $Y$, et al. Subcutaneous panniculitis-like T-cell lymphoma: successful initial treatment with prednisolone and cyclosporin A. Intern Med 2006; 45: $21-4$

34. Chen R, Liu L, Liang YM. Treatment relapsed subcutaneous panniculitis-like T-cell lymphoma together HPS by Cyclosporin A. Hematol Rep 2010; 26: e9.

35. Shen G, Dong L, Zhang S. Subcutaneous panniculitis-like T cell lymphoma mimicking early-onset nodular panniculitis. Am J Case Rep 2016; 17: 429-33.

36. Ichii $M$, Hatanaka $K$, Imakita $M$, Ueda $Y$, Kishino $B$, Tamaki $T$. Successful treatment of refractory subcutaneous panniculitis-like T-cell lymphoma with allogeneic peripheral blood stem cell transplantation from HLA-mismatched sibling donor. Leuk Lymphoma 2006; 47: 2250-2.

37. Alaibac $M$, Berti $E$, Pigozzi $B$, et al. High-dose chemotherapy with autologous blood stem cell transplantation for aggressive subcutaneous panniculitis-like T-cell lymphoma. J Am Acad Dermatol 2005; 2: S121-3. 